\title{
MiR-I39-5p-ZEBI is a Molecular Regulator of Growth, Invasion, and Epithelial-to-Mesenchymal Transition of Cervical Cancer
}

This article was published in the following Dove Press journal: Cancer Management and Research

Jinrui Sun'

Shanshan Wang ${ }^{2}$

Ping Liu'

Yulan $\mathrm{Liu}^{3}$

'Department of Gynecology, Shanxi Provincial People's Hospital, Taiyuan, Shanxi Province 030012, People's Republic of China; ${ }^{2}$ Department of Cardiology, Yidu Central Hospital of Weifang City, Weifang, Shandong, People's Republic of China; ${ }^{3}$ Department of Gynecology, Maternal and Child Health Hospital of Hubei Province, Wuhan, Hubei 430070, People's Republic of China
Objective: To verify that miR-139-5p-zinc finger E-box-binding homeobox 1 (ZEB1) is a molecular regulator of the biological function and epithelial-mesenchymal transition (EMT) of cervical cancer (CC) cells.

Methods: Cancerous tissues, corresponding paracancerous tissues, and serum were sampled from patients with CC. MiR-139-5p and ZEB1 in tissue specimens, serum specimens, and purchased CC cell lines were quantified, and Pearson correlation coefficient was adopted for correlation analysis of $m i R-139-5 p$ in clinical specimens. Receiver operating characteristic (ROC) curves were adopted to analyze the diagnostic value of $m i R-139-5 p$ and ZEB1 for CC. The expression of genes in CC cells was changed by transfection. The proliferation, colony formation, invasion, and apoptosis of cells were determined, and the protein level of EMT markers (N-cadherin, vimentin, and E-cadherin) was also quantified. Moreover, the targeting relationship between $m i R-139-5 p$ and ZEB1 was determined.

Results: Our data showed that the expression of $m i R-139-5 p$ decreased greatly in $\mathrm{CC}$ tissues, and it also significantly decreased in the serum, while the expression of serum $Z E B 1$ was opposite. In addition, the $m i R-139-5 p$ expression in CC tissues was positively correlated with that in serum, while serum $m i R-139-5 p$ was negatively correlated with serum $Z E B 1$. The areas under the curves (AUCs) of the two for identifying CC were 0.923 and 0.890 , respectively. Both up-regulation of $m i R-139-5 p$ and down-regulation of ZEB1 suppressed the colony formation, proliferation, invasion, and EMT of CC cells, and intensified their apoptosis. Moreover, miR-139-5p negatively regulated the transcription of $Z E B 1$, and down-regulation of the former could reverse the molecular regulatory effects of downregulating $Z E B 1$ on the above biological behaviors of $\mathrm{CC}$ cells.

Conclusion: The above data imply that $m i R-139-5 p-Z E B 1$ axis may be the key to curbing the progression of $\mathrm{CC}$.

Keywords: cervical cancer, miR-139-5p, ZEB1, growth, invasion, epithelial-mesenchymal transition

\section{Introduction}

Cervical cancer (CC) is a gynecologic tumor with poor prognosis, which may deteriorate to brain metastasis or bone metastasis. ${ }^{1,2}$ According to global data, there were 570,000 new cases with CC and up to 311,000 cases dying of the disease in $2018 .^{3}$ At present, the exact mechanism of the progression of $\mathrm{CC}$ remains unclear, and biological indicators for the diagnosis of $\mathrm{CC}$ remain to be found, ${ }^{4,5}$ so it is crucial for the management of $\mathrm{CC}$ to study the mechanism of $\mathrm{CC}$ progression and find potential screening indicators.
Department of Gynecology, Maternal and Child Health Hospital of Hubei Province, NO. 745 Wuluo Road, Hongshan, Wuhan, Hubei 430070, People's Republic of China Tel +86-I5698784852

Email yulantang885@163.com 
MicroRNA (miRNA), as a small non-coding RNA molecule, is a molecular regulator of the growth, invasion and epithelial-mesenchymal transition (EMT) of various tumors including $\mathrm{CC}^{6-8}$ MiRNA is found to have regulatory effect in various gynecologic tumors. For example, in endometrial carcinoma, miR-136 regulates the development of tumor cells by regulating NOTCH3 under the regulation of Circ pumilio homolog 1 (PUM1), ${ }^{9}$ and miR-653-5p accelerates the malignant growth of $\mathrm{CC}$ under the sponge of DGUOK-AS1. ${ }^{10}$ In the present study, we focused on the expression and role of $m i R-139-5 p$ in $\mathrm{CC}$, which reportedly serves as a tumor inhibitor in the disease. ${ }^{11}$ It has been found that miR-139-5 $p$ can regulate the pathological processes of glioma such as metastasis, infiltration, and EMT. ${ }^{12}$ We found a potential targeting relationship between $m i R-139-5 p$ and zinc finger E-boxbinding homeobox 1 (ZEB1) based on an online target gene prediction website. Moreover, it has been uncovered that both miR-139-5p and ZEB1 have targeted regulatory effects on breast cancer (BC), colorectal cancer (CRC), as well as glioblastoma. For example, $m i R-139-5 p-Z E B 1$ axis affects the malignant biological behaviors of $\mathrm{BC}$ cells under the regulation of $T T N-A S 1$, and it also affects the pathological changes of CRC cells under the regulation of HLA complex P5 (HCP5). ${ }^{13-15}$ However, ZEB1 is reported abnormally up-regulated in cases with $\mathrm{CC}$, which affects the proliferation, metastatic behavior, and EMT of $\mathrm{CC}$ cells. ${ }^{16,17}$

In the present study, we inferred that miR-139-5p-ZEBI was a molecular regulator of the growth, invasion, and EMT of CC, and thus we conducted relevant studies to verify it.

\section{Materials and Methods Sample Collection}

This study was approved by the Ethics Committee of Shanxi Provincial People's Hospital, and written consent forms were obtained from all participants. Cancerous tissues and corresponding paracancerous tissues were sampled from99 patients with CC (CC group) during operation from April 2016 to April 2020, and serum was also sampled from them before operation. All the patients were diagnosed with $\mathrm{CC}$ based on pathology. ${ }^{18}$ In addition, serum was sampled from 50 healthy individuals in physical examination during the same period as a healthy control group (HC group). All patients with $\mathrm{CC}$ had not received any surgery or drug treatment before sample collection, and all sampled specimens were stored at $-80^{\circ} \mathrm{C}$ for later analysis. Moreover, the clinical staging of the patients was evaluated according to the International Federation of Gynecology and Obstetrics (FIGO) criteria, ${ }^{19}$ and the histological grading of them is also evaluated in Table 1.

\section{Cell Culturing}

Human normal cervical epithelial cells (HUCEC) (FE1528, Qiming Biotechnology Co., Ltd., Shanghai, China) and human CC cells (HeLa 229, H1HeLa, C-33 $\mathrm{A}$, and MS751) (YBCC102035, YBCC101654, YBCC101021, and YBCC100277, Yubo Biological Technology Co., Ltd., Shanghai, China) were incubated in dulbecco's modified eagle medium (DMEM; PM150220B, Yaji Biotechnology Co., Ltd., Shanghai, China) supplemented with $10 \%$ phosphate buffer saline (PBS) under $5 \% \mathrm{CO}_{2}$ at $37^{\circ} \mathrm{C}$.

\section{Cell Transfection}

Transfectants used in this study included miR-139-5p mimics (miR-139-5p), inhibition sequence (inhibitor), $\mathrm{miR}$ negative control (miR-NC), targetedly overexpressed sequence of $Z E B 1$ (ZEB1), targetedly inhibited sequence of $Z E B 1$ (si-ZEB1), and si-negative control (si-NC), which were mainly purchased from Shanghai Huishen Biological Technology Co., Ltd. CC cells were transfected with above transfectants, respectively using a cell transfection kit (YSRIBIO-C5838, Yansheng Industrial Co., Ltd., Shanghai, China) in strict accordance with the kit instructions. After $48 \mathrm{~h}$, the transfected cells were harvested for later analysis.

\section{Real-Time Polymerase Chain Reaction (PCR)}

Total RNA was extracted from harvested tissues, serum, as well as cells by a TRIzol kit (R523-200, Spectral Experimental Equipment Technology Co., Ltd., Dongguan, China), followed by detection of its purity and concentration with an ultraviolet spectrophotometer (SPCC, Spectral Experimental Equipment Technology Co., Ltd., Dongguan, China). Subsequently, total RNA was reversely transcribed into cDNA and amplified. The data in this assay were analyzed using the $2^{-\Delta \Delta \text { ct }}$, with $\beta$ Actin as internal reference for ZEB1, Caspase-3, Bax, and $B c l-2$, and $U 6$ as internal reference for miR-139-5p. 
Table I Relationship Between MiR-I39-5p and Pathological Data of Patients with CC [Mean \pm SD]

\begin{tabular}{|c|c|c|c|c|}
\hline Factor & $n=76$ & $M i R-I 39-5 p$ & T-value & P-value \\
\hline Menopause or not? & & & 0.509 & 0.612 \\
\hline No & 32 & $3.72 \pm 0.55$ & & \\
\hline Yes & 44 & $3.65 \pm 0.62$ & & \\
\hline Age & & & 0.892 & 0.376 \\
\hline$<55$ years old & 38 & $3.75 \pm 0.61$ & & \\
\hline$\geq 55$ years old & 38 & $3.62 \pm 0.66$ & & \\
\hline FIGO staging & & & 6.204 & $<0.001$ \\
\hline $\mathrm{I} / \mathrm{II}$ & 36 & $4.10 \pm 0.51$ & & \\
\hline $\mathrm{III} / \mathrm{IV}$ & 40 & $3.22 \pm 0.70$ & & \\
\hline Pathological differentiation & & & 3.968 & $<0.001$ \\
\hline Low differentiation & 42 & $3.38 \pm 0.63$ & & \\
\hline Moderate and high differentiation & 34 & $3.90 \pm 0.48$ & & \\
\hline Tumor diameter & & & 0.887 & 0.378 \\
\hline$<4 \mathrm{~cm}$ & 41 & $3.7 I \pm 0.64$ & & \\
\hline$\geq 4 \mathrm{~cm}$ & 35 & $3.59 \pm 0.52$ & & \\
\hline Histological type & & & 0.925 & 0.358 \\
\hline Squamous cell carcinoma & 48 & $3.73 \pm 0.65$ & & \\
\hline Adenocarcinoma & 28 & $3.60 \pm 0.47$ & & \\
\hline SCC-Ag (ng/mL) & & & 1.150 & 0.254 \\
\hline$<1.5$ & 26 & $3.8 I \pm 0.4 I$ & & \\
\hline$\geq 1.5$ & 50 & $3.63 \pm 0.74$ & & \\
\hline
\end{tabular}

Abbreviations: miR, microRNA; CC, cervical cancer; FIGO, Federation of Gynecology and Obstetrics; SCC-Ag, squamous cell carcinoma antigen.

\section{Western Blot}

Protein in tissues or cells was isolated by RIPA buffer (HLIT0050, Haling Biotechnology Co., Ltd., Shanghai, China), and then ionized and transferred to a membrane. Subsequently, the membrane was sealed with blocking solution (BH-DB6564, Bohu Biotechnology Co., Ltd., Shanghai, China) for $1 \mathrm{~h}$, followed by incubation with primary antibodies (N-cadherin, vimentin, E-cadherin, $Z E B 1$, and $\beta$-Actin) at $4{ }^{\circ} \mathrm{C}$ overnight. Afterwards, the membrane was subjected to 1-h incubation with secondary antibody at $4^{\circ} \mathrm{C}$. All antibodies were purchased from Shanghai Xuanya Biotechnology Co., Ltd. Finally, the protein strips were visualized and analyzed by Quantity One software (ECL-0013, EASYBIO Technology Co., Ltd., Beijing, China).

\section{Cell Proliferation}

Cell proliferation was determined using a MTT kit $(\mathrm{RF}(\mathrm{m})$ 11,473, Qiming Biotechnology Co., Ltd., Shanghai, China) as follows: The cells were seeded into a 96-well plate at 1000 cells $/$ well, incubated at $37^{\circ} \mathrm{C}$ for $3 \mathrm{~d}$, and then added with $20 \mu \mathrm{L}$ MTT solution $(2.5 \mathrm{mg} / \mathrm{mL})$, followed by 3-h incubation. Subsequently, each well was added with $150 \mu \mathrm{L}$ dimethyl sulfoxide, and the optical density of cells in each group at $570 \mathrm{~mm}$ was detected using an ELx808LBS microplate reader (ZEPING Bioscience \& Technologies Co., Ltd., Beijing, China).

\section{Colony Formation Assay}

$\mathrm{CC}$ cells $\left(2 \times 10^{3}\right.$ cells $)$ were seeded into a 6 -well plate. After14 d, the cells were immobilized with $4 \%$ paraformaldehyde (PFA; M002, Gefan Biotechnology Co., Ltd., Shanghai, China) at room temperature for 10-20 minutes, and then dyed with $0.05 \%$ crystal violet for $10-20 \mathrm{~min}-$ utes. Finally, colonies were counted under a microscope.

\section{Cell Invasion}

Collected cells were transferred to a upper compartment coated with Matrigel (356,234, Haoyang Biotechnology Co., Ltd., Shanghai, China), and medium with $20 \%$ FBS was added into the lower compartment. The insert was incubated at $37^{\circ} \mathrm{C}$ for $24 \mathrm{~h}$, and then the cells were immobilized with PFA for 10 min after being washed with PBS 
three times. Then the cells were dyed with $0.5 \%$ crystal violet. Finally, invasive cells were counted under a microscope.

\section{Cell Apoptosis}

Cell apoptosis was detected by a cell apoptosis kit (BW3302, Biomiga Medical Technology Co., Ltd., Hangzhou, China) as follows: The transfected cells were trypsinized, and then the cells were washed with PBS twice, and then mixed with $100 \mu \mathrm{L}$ binding buffer to produce $1 * 10^{6}$ cells $/ \mathrm{mL}$ suspension. The suspension was mixed with $10 \mu \mathrm{L}$ AnnexinV-FITC and $10 \mu \mathrm{L}$ PI in sequence, cultured at room temperature in the dark for 5 min, and finally determined with a DxFLEX flow cytometer (Beckman Coulter Trading (China) Co., Ltd., Shanghai, China).

\section{Luciferase Determination}

Cells were transferred to a 24-well plate. After $24 \mathrm{~h}, Z E B 1$ wild-type (ZEB1-Wt) and ZEB1 mutant (ZEB1-Mut) were constructed by a dual luciferase reporter (DLR) gene assay kit (SLDL-100, Bei Nuo Biotechnology Co., Ltd., Shanghai, China) and transfected into cells. After $48 \mathrm{~h}$, the luciferase activity of the cells was determined.

\section{Xenotransplantation Tumor Model of Mouse}

We purchased 12 female BALB/c nu/nu nude mice (4 weeks old and 18-22 $\mathrm{g}$ in weight) from Cavens Experimental Animal Co., Ltd., Changzhou, China, and raised them in specific pathogen-free conditions under 12 $\mathrm{h}$ light $/ 12 \mathrm{~h}$ dark cycle and studied them. The animal study was approved by the Animal Ethics Committee of our hospital (Shanxi Provincial People's Hospital), and it was carried out in strict accordance with the Laboratory animal - Guideline for ethical review of animal welfare (Standardization Administration of the People's Republic of China) for protecting animals. First, the mice were fed for 1 week, and then randomly divided into two groups (MOCK group and miR-139-5p group). H1HeLa cells transfected with miR-NC or miR-139-5p mimics (107 cells per mouse) were injected subcutaneously into the left armpit of mice in the two groups. On the 21st day, mice were anesthetized with chloral hydrate (A4988, Shifeng Biological Technology Co., Ltd., Shanghai, China) and euthanized (decapitated), and their tumors were removed, measured and weighed.

\section{Statistical Analyses}

In the present study, all assays were repeated three times and the collected data were analyzed statistically and visualized into figures via GraphPad 6. Data differences were analyzed using the independent $t$-test, one-way ANOVA, LSD- $t$-test, and Bonferroni post hoc test. In addition, receiver operating characteristic (ROC) curves were drawn to analyze the area under the curve (AUC) of miR-139-5p in diagnosing CC, and Pearson correlation analysis was carried out to analyze the relationship between miR-139-5p expression in the serum of patients with $\mathrm{CC}$ and that in cancerous tissues of the patients, and the correlation between serum miR-139-5p and serum ZEB 1. $P<0.05$ indicates a remarkable difference.

\section{Results}

\section{MiR-I 39-5p Was Down-Regulated in Cancerous Tissues and Serum of Patients with CC, While ZEBI Was Up-Regulated in Them}

For the purpose of verifying our assumption, we quantified $m i R-139-5 p$ in the cancerous tissues and serum specimens from patients with $\mathrm{CC}$, finding that $m i R-139-5 p$ was lowly expressed in both specimens. The results implied that $m i R$ $139-5 p$ may serve as a cancer suppressor in CC. Further investigation revealed that the miR-139-5p expression in the two specimens was positively correlated, suggesting that $m i R-139-p$ may have a certain clinical value as a noninvasive serum indicator. We also found that the expression of ZEB1 in the two samples was significantly opposite to that of $m i R-139-5 p$ in them, and the expression of $m i R$ $139-5$ and $Z E B 1$ in serum was significantly negatively correlated. Then, we drew ROC curves of $m i R-139-p$ and $Z E B 1$ in identifying $C C$ as serum indicators, and found that the AUC of miR-139-5 for diagnosing $\mathrm{CC}$ was as high as 0.923 , while that of $Z E B 1$ was 0.890 , indicating that they may be used as auxiliary indicators for $\mathrm{CC}$ screening Figure 1.

\section{MiR-I 39-5p Was Linked to the FIGO Staging and Pathological Differentiation of Patients with CC}

With the aim of exploring the potential correlation between $m i R-139-5 p$ and pathological parameters ofCC patients, we analyzed the relative expression of $m i R-139-5 p$ in patients with different pathological parameters, finding that $m i R$ - 

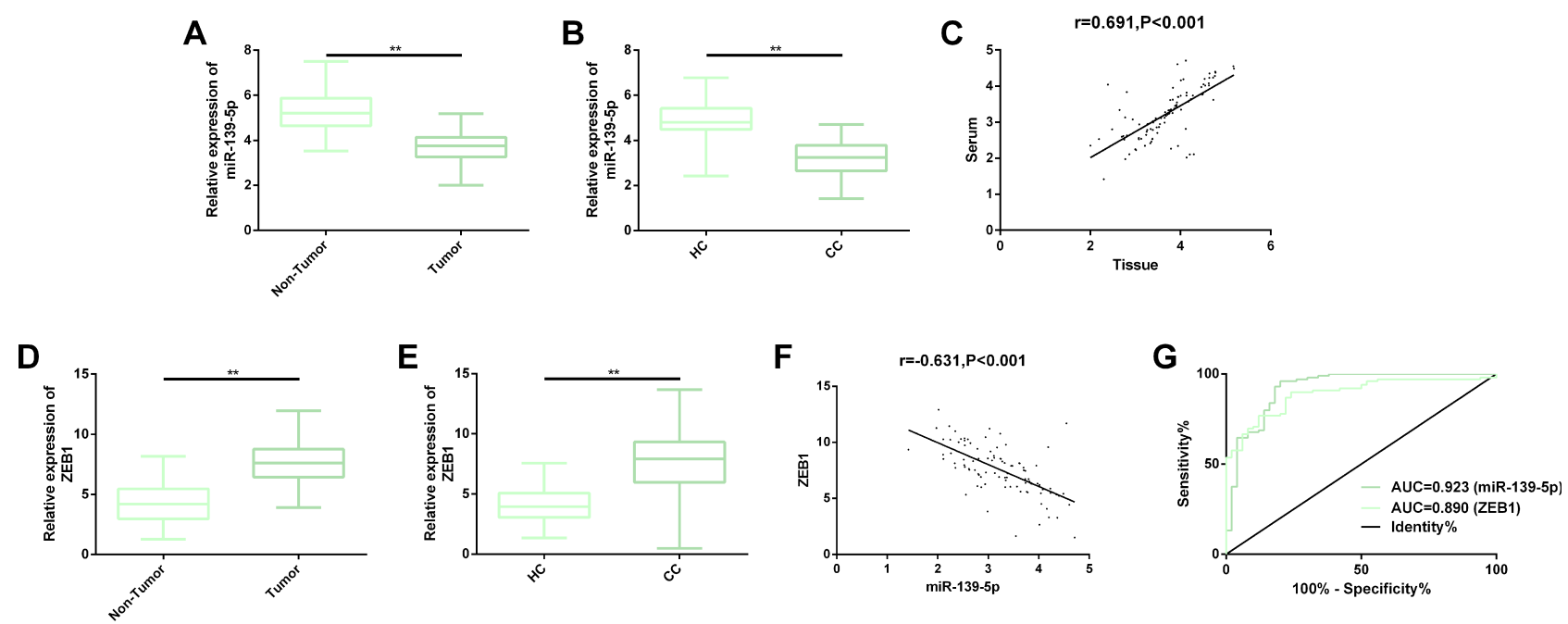

Figure I Expression of miR-I39-5p and ZEBI in the cancerous tissues and serum of patients with CC. (A, B) MiR-I39-5p was down-regulated in both cancerous tissues and serum of patients with CC. (C) The expression of miR-I39-5p in cancerous tissues was strongly positively correlated with that in serum. (D, E) The expression of ZEBI was up-regulated in cancerous tissues and serum of patients with CC. (F) MiR-I39-5p and ZEBI in serum were negatively correlated. (G) AUC of serum miR-I39-5p in identifying CC exceeded 0.900 , and that of serum ZEBI for identifying CC exceeded 0.850 .

Note: **Indicates that in terms of inter-group comparison, $P<0.01$.

Abbreviations: miR, microRNA; CC, cervical cancer; AUC, area under the curve; HC, healthy control; ZEBI, zinc finger E-box-binding homeobox I.

$139-5 p$ was not strongly linked to menopause, age, tumor diameter, histological grading, as well as squamous cell carcinoma antigen (SCC-Ag) (all $P>0.05$ ), but low $m i R$ 139-5p expression was significantly linked to high FIGO staging and low differentiation (both $P<0.05$ ). The results imply that $m i R-139-5 p$ may be helpful to predict FIGO staging and differentiation of patients in Table 1.

\section{MiR-I39-5p Could Inhibit the Growth, Invasion, and EMT of CC Cells}

In order to explore the potential anticancer effect of $m i R$ $139-5 p$ in $\mathrm{CC}$, we analyzed its effect on the biological behaviors of $\mathrm{CC}$ cells. First of all, we found that $m i R$ 139-5p was generally expressed at low levels in CC cells, especially in H1HeLa and C-33 A cells, so we mainly studied $\mathrm{H} 1 \mathrm{HeLa}$ and $\mathrm{C}-33 \mathrm{~A}$ cells. We transfected $m i R$ 139-5p mimics and miR-139-5p inhibition sequence into H1HeLa and C-33 A cells to overexpress and underexpress $m i R-139-5 p$ in them, respectively. The cellular function test revealed that down-regulating miR-139-5p accelerated the malignant proliferation, invasion, colony formation, and EMT of CC cells, inhibited the apoptosis rate, decreased the levels of pro-apoptotic factors (Caspase-3 and Bax), and increased the level of antiapoptotic factor $(B c l-2)$, but the above results were significantly reversed after $m i R-139-5 p$ was up-regulated: The malignant behaviors of CC cells were strongly suppressed and the cell apoptosis was induced. The results implied that regulating miR-139-5p could control the malignant development of CC cells, and miR-139-5p had certain cancer inhibiting effect in CC Figure 2.

\section{MiR-I39-5p Negatively Affected the Transcription and Protein Level of ZEB I}

For the purpose of further understanding the mechanism of miR-139-5p in CC, we also analyzed its potential downstream targets. Based on TargetScan (http://www.targets can.org/vert 72/), we found potential binding locus between miR-139-5p and ZEB1, so we carried out analysis for further verification. The DLR revealed that $m i R-139-5 p$ mimics down-regulated only ZEB1-Wt (not ZEB1-Mut), and the Western blot assay showed that miR-139-5p negatively regulated the transcription and protein level of $Z E B 1$, which suggested that miR-139-5p could regulate ZEB1 negatively (Figure 3).

\section{ZEBI Was Up-Regulated in CC Cells and Could Promote the Growth, Invasion, and EMT of CC Cells}

We also explored whether $Z E B 1$ had influence on the malignant function of $\mathrm{CC}$ cells. First of all, we quantified ZEB1 in CC cells, and found that it was generally abnormally up-regulated, indicating that it may also be involved in the malignant development of CC cells. Further analysis of cell behaviors showed that up-regulation of ZEBI 
A

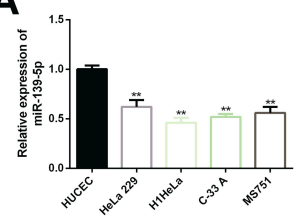

D
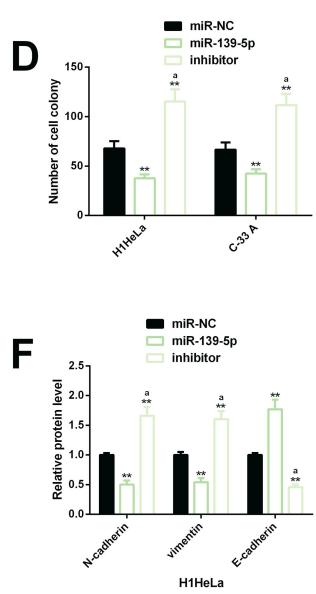

H

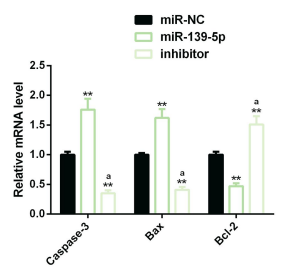

B
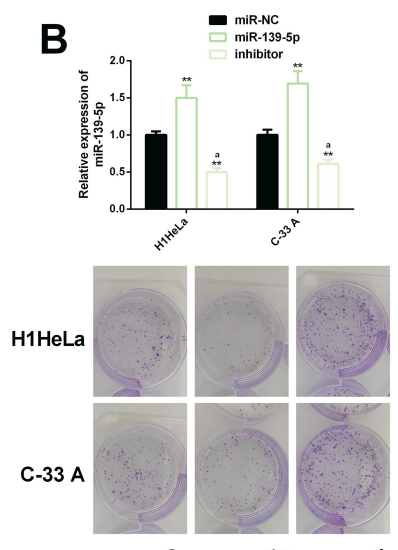

miR-NC miR-139-5P inhibitor

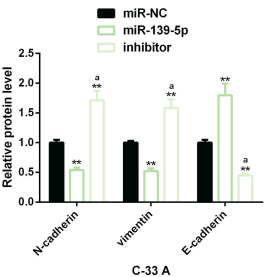

G
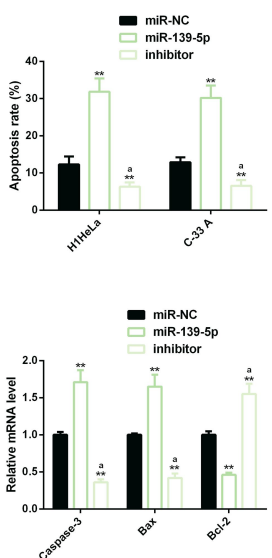

C
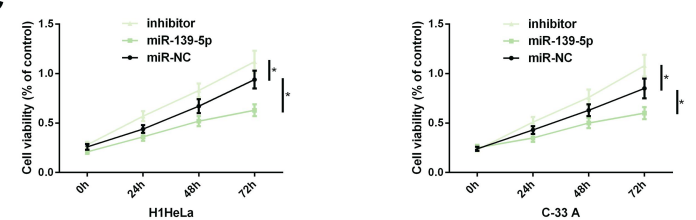

$\mathbf{F}$
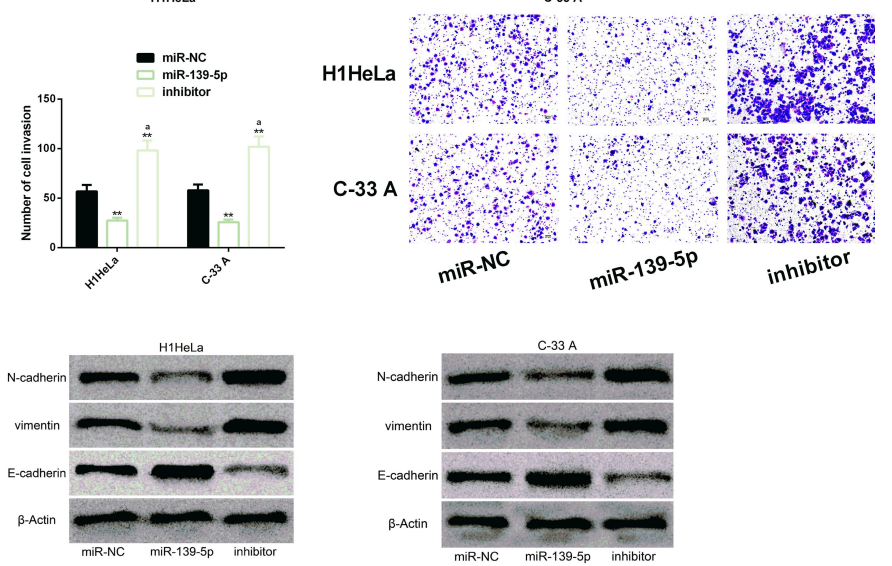

C-33 A
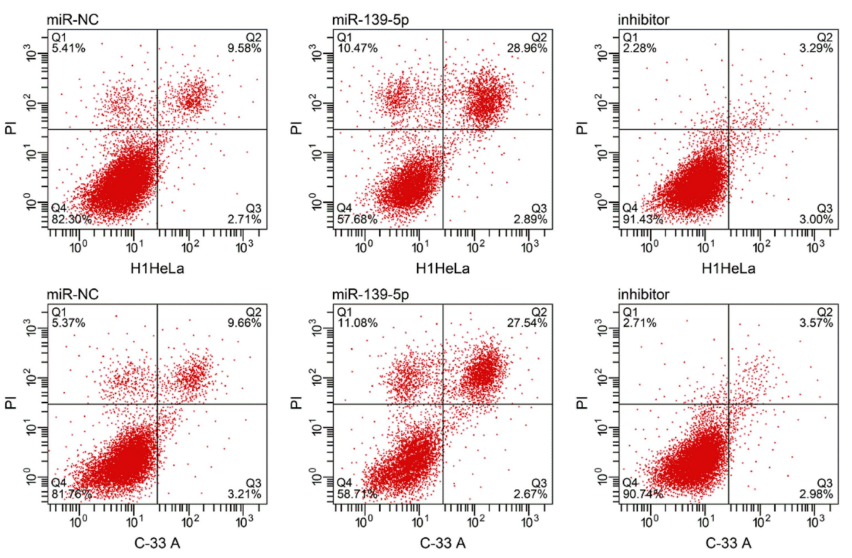

Figure 2 Effects of miR-139-5p on the biological function of CC cells. (A) MiR-I39-5p was significantly down-regulated in CC cells. (B) Transfection efficiency of miR-I39-5p. (C-F) Up-regulation of miR-I39-5p significantly inhibited the malignant proliferation, colony formation, invasion, EMT of CC cells. Protein profiling of EMT-related factors of CC cells. (G) Up-regulation of miR-I39-5p significantly promoted the apoptosis rate of CC cells. Its cell flow cytometry profiling. (H) Down-regulation of ZEBI increased the expression of Caspase-3 and Bax, but inhibited the expression of BCl-2.

Notes: In terms of inter-group comparison or comparison with miR-NC, *Indicates $P<0.05$ and **Indicates $P<0.01$; in terms of comparison with miR-I39-5p, ${ }^{a}$ Indicates $P<0.01$ in terms of comparison with miR-I39-5p.

Abbreviations: miR, microRNA; CC, cervical cancer; EMT, epithelial-mesenchymal transition; NC, negative control; PI, propidium iodide.

promoted the malignant proliferation, colony formation, invasion, and EMT of CC cells, strongly inhibited cell apoptosis, decreased the expression of Caspase-3 and $B a x$, and increased the expression of $B c l-2$, while knockdown of $Z E B 1$ significantly inhibited the malignant function of $\mathrm{CC}$ cells and intensified their apoptosis. The results implied that both down-regulating $Z E B 1$ and up-regulating miR-139-5p had significant anti-tumor effects on CC cells (Figure 4).

\section{Down-Regulating MiR-I39-5p Could} Weaken the Anti-Tumor Activity of Transfecting Si-ZEBI in CC Cells

We carried out a co-transfection experiment about miR-139$5 p$ and $Z E B 1$, finding that down-regulating miR-139-5p could reverse the inhibiting effect of down-regulating $Z E B 1$ on the malignant proliferation, invasion, colony formation, as well as EMT of CC cells and induction of it on the cell apoptosis (including the effect on apoptotic factor), 


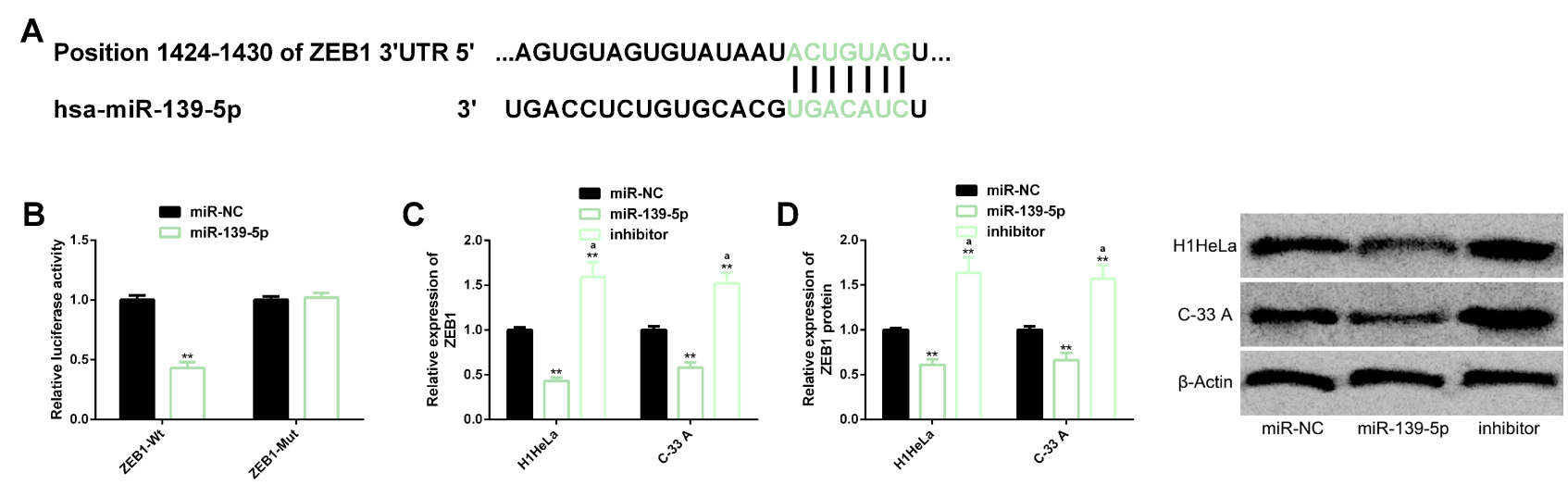

Figure 3 Targeting regulatory relationship between miR-I39-5p and ZEBI. (A) Binding locus between miR-I39-5p and ZEBI. (B) DLR result. (C, D) Up-regulation of miR139-5p down-regulated the transcription and protein level of $Z E B I$. Its protein profiling.

Notes: **Indicates $P<0.01$ in terms of comparison with the miR-NC group. ${ }^{a}$ Indicates $P<0.01$ in terms of comparison with the miR-139-5P group.

Abbreviations: miR, microRNA; ZEBI, zinc finger E-box-binding homeobox I; DLR, dual luciferase reporter; NC, negative control; Wt, wild type; Mut, mutant.

which implied that miR-139-5p regulated the biological behaviors of CC cells through targeting ZEB1 (Figure 5).

\section{Up-Regulation of MiR-I39-5p Can Inhibit Tumor Growth in CC Xenotransplantation Model Mice}

To study the effect of miR-139-5p on the tumor growth of $\mathrm{CC}$ model in vivo, H1HeLa cells transfected with miR-NC or $m i R-139-5 p$ mimetic were inoculated into the left armpit of nude mice. The results showed that at 4 weeks after inoculation, up-regulation of $m i R-139-5 p$ significantly inhibited the tumor volume and mass in CC xenotransplantation model mice compared with the MOCK group (Figure 6).

\section{Discussion}

The etiology of $\mathrm{CC}$ is strongly linked to human papillomavirus (HPV) infection. As a fatal gynecologic tumor, $\mathrm{CC}$ shows a continuously increasing incidence in some countries, which results in great cancer burden to women. ${ }^{20,21}$ At present, $\mathrm{CC}$ is intractable, which is mainly manifested in drug resistance and lack of effective treatment strategies for advanced or recurrent $\mathrm{CC}{ }^{22} \mathrm{~A}$ large body of evidence has verified that the abnormal dysregulation of miRNA is linked to tumor progression, ${ }^{23,24}$ so analyzing the molecular mechanism of miRNA in CC is of great value for improving treatment schemes, prevention, and management of CC.

According to clinical analysis, $m i R-139-5 p$ was expressed at low levels in cancerous tissues and serum of patients with CC, while ZEBI was expressed at high levels in them, and both serum $m i R-139-5 p$ and ZEB1 had relatively high diagnostic value $(\mathrm{AUC}=0.923$ and 0.890 , respectively). Therefore, we believed that the two may have good diagnostic value in CC., and the former may inhibit cancer, while the latter may induce cancer. One study by Miyoshi et $\mathrm{al}^{25}$ has pointed out that serum miR$139-5 p$ in patients with CRC is helpful to predict tumor recurrence and metastasis. In this study, further analysis showed that low miR-139-5p level was strongly linked to high FIGO staging and low pathological differentiation, indicating that $m i R-139-5 p$ level also has certain predictive value for pathological parameters of patients. According to one report by Wang et al, ${ }^{26}$ low miR-139-5p level is linked to FIGO staging and lymph node metastasis of patients with ovarian cancer, which is different from the results of our study. Similarly, one study by Ji et $\mathrm{al}^{11}$ has revealed that the low $m i R-139-5 p$ level was strongly linked to lymph node metastasis of patients with CC. We inferred that $m i R-139-5 p$ may also be adopted to predict the prognosis of patients, but we did not get data about it in this study, so we were unable to further verify it. In addition to cases with CC, $m i R-139-5 p$ is also lowly expressed in cases with non-small cell lung cancer (NSCLC), lowgrade glioma in children, as well as prostate cancer, implying that $m i R-139-5 p$ may have relatively extensive cancer inhibition performance. ${ }^{27-29}$ For example, it exerts anticancer effect in NSCLC by inhibiting oncogenic gene, c-Met, in a targeted manner, and suppresses the survival of low-grade glioma cells in children by disabling oncogenic signaling pathway conduction (PI3K-AKT pathway) in them. In addition, it also hinders the progression of prostate cancer by lowering SOX5. On the other hand, 
A

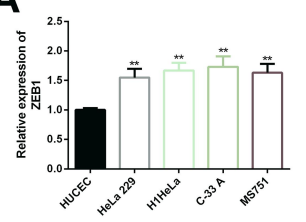

D

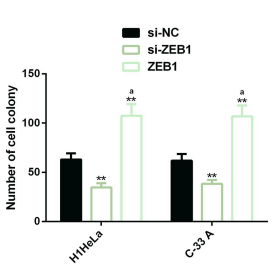

F

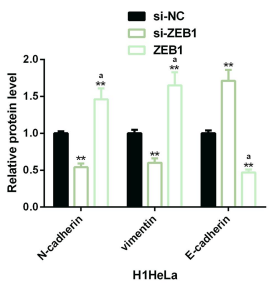

H

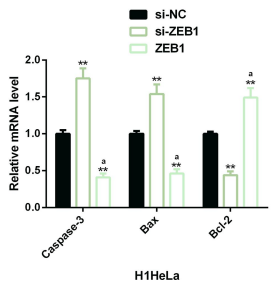

B
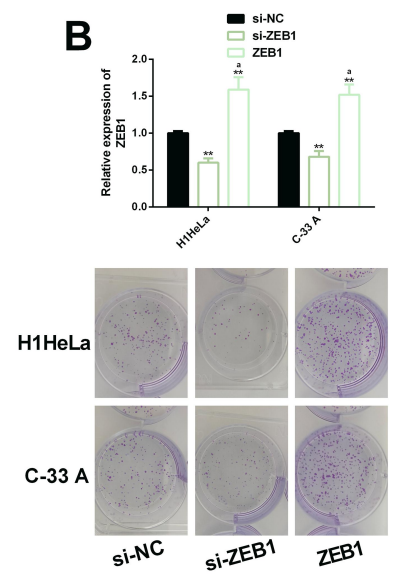

si-NC

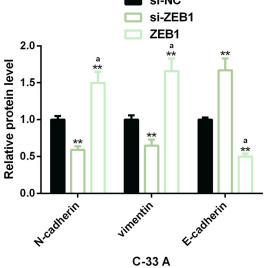

G
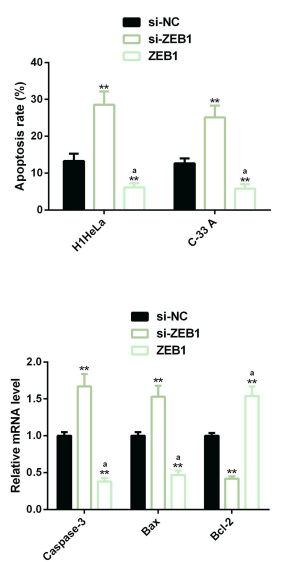

C

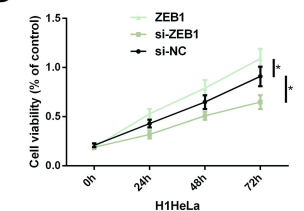

E
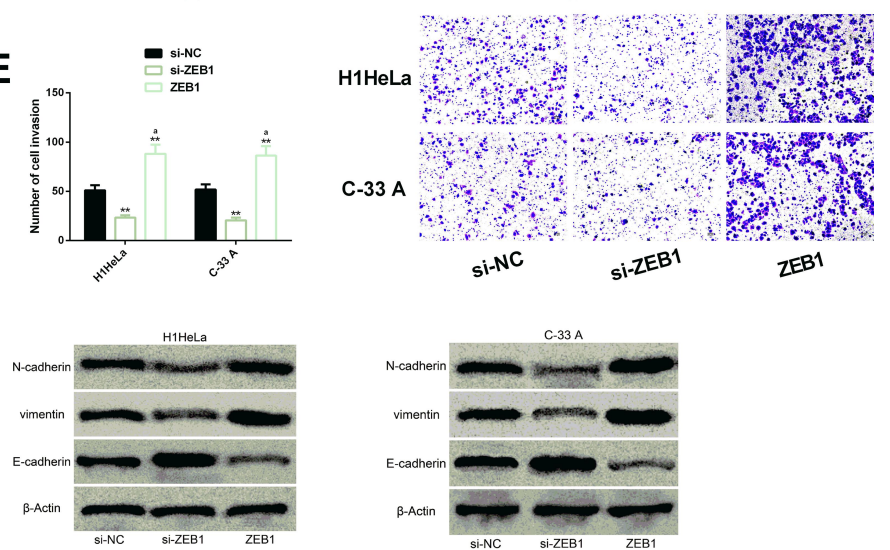
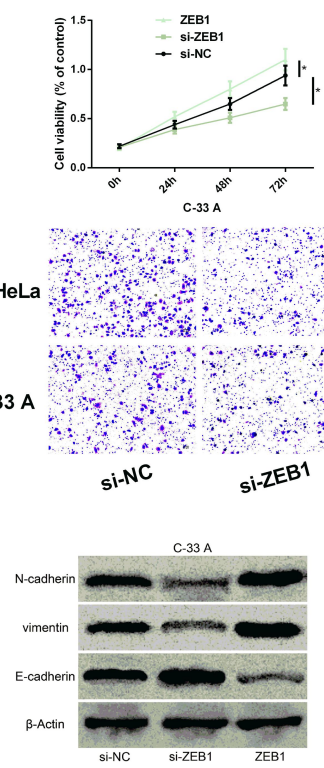
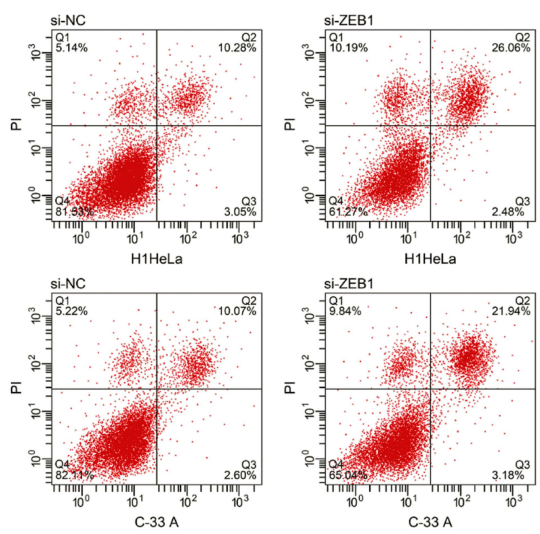
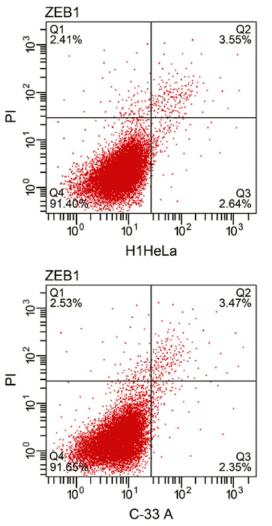

Figure 4 Effects of ZEBI on the biological function of CC cells. (A) ZEBI was generally highly expressed in CC cells. (B) Transfection efficiency of ZEBI. (C-F) Downregulation of $Z E B I$ significantly inhibited the malignant proliferation, colony formation, invasion, EMT of CC cells. Protein profiling of EMT-related factors. (G) Downregulation of ZEBI significantly promoted the apoptosis rate of CC cells. Its cell flow cytometry profiling. (H) Down-regulation of ZEBI increased the expression of Caspase3 and Bax, but inhibited the expression of $\mathrm{Bcl}-2$.

Notes: In terms of inter-group comparison or comparison with si-NC, *Indicates $P<0.05$ and **Indicates $P<0.0 \mathrm{I}$; in terms of comparison with $Z E B I$, ${ }^{a}$ Indicates $P<0.0$ I Abbreviations: ZEBI, zinc finger E-box-binding homeobox I; CC, cervical cancer; EMT, epithelial-mesenchymal transition; si, short interfering; NC, negative control; PI, propidium iodide; Bax, Bcl-2-associated $X$ protein; Bcl-2, B-cell lymphoma-2.

ZEB1 is not only highly expressed in cases with CC, but also overexpressed in cases with diseases such as esophageal squamous cell carcinoma, liver cancer, and cutaneous squamous cell carcinoma, which suggests that it may have extensive carcinogenicity. ${ }^{30-32}$

An increasing number of scholars have shown interest in studying the influence of miR-139-5p on gynecologic tumors, and have published many studies. For instance, Liu et $\mathrm{a}^{33}$ have reported that miR-139-5p can suppress the viability and migration of endometrial carcinoma cells through targeted inhibition on HOXA10. Liu et $\mathrm{al}^{34}$ have pointed out that $m i R-139-5 p$ can suppress the progression of ovarian cancer by regulating downstream target, ROCK2, under the targeted control of TTN-ASI. Furthermore, Ji et $\mathrm{al}^{11}$ have verified that $m i R-139-5 p$ can mediate TCF4 and inactivate the $W n t / \beta$-catenin signal, thereby exerting potential therapeutic effect on CC. In our study, miR-139$5 p$ exerted its anticancer ability in $\mathrm{CC}$ by inhibiting the proliferation, colony formation, invasion, and EMT of CC cells and intensifying their apoptosis, increasing the 
A

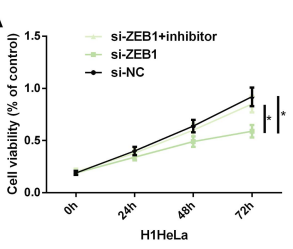

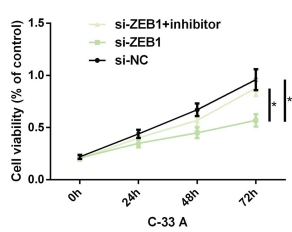
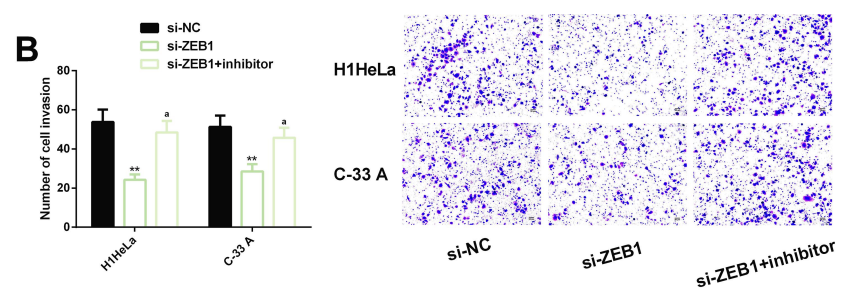
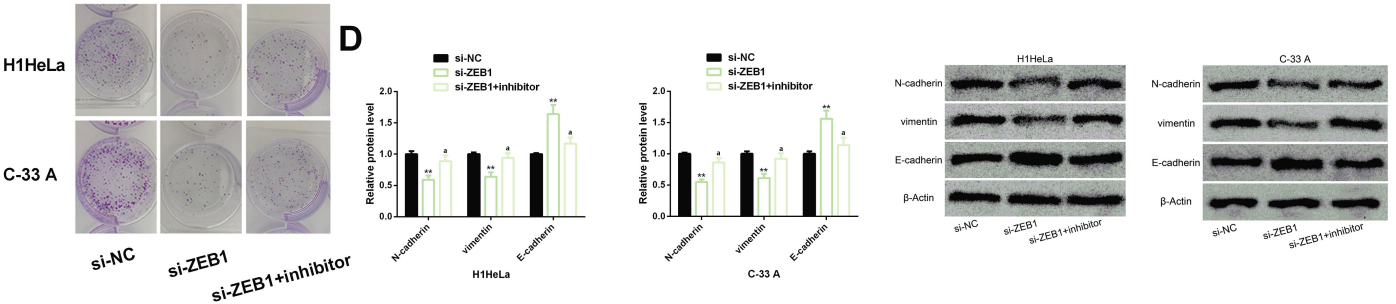

E
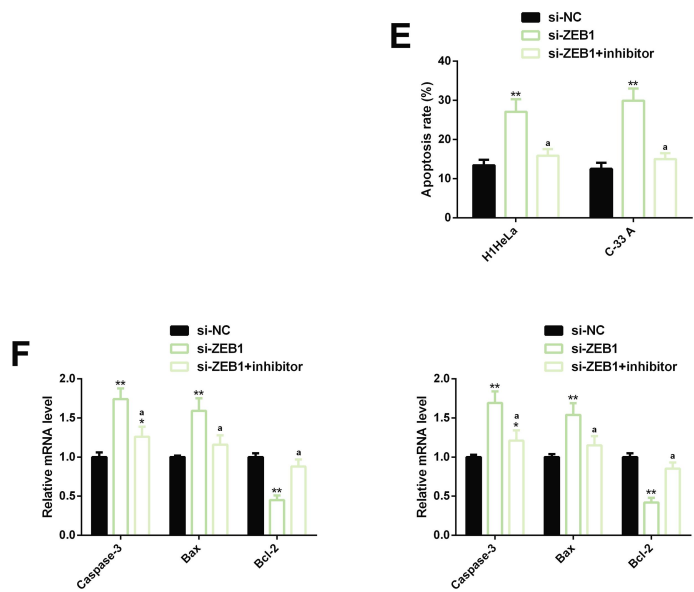

H1HeL

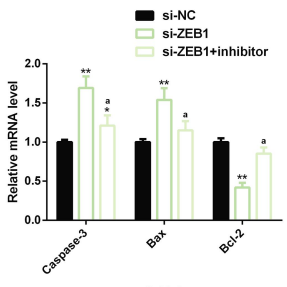

C-33A
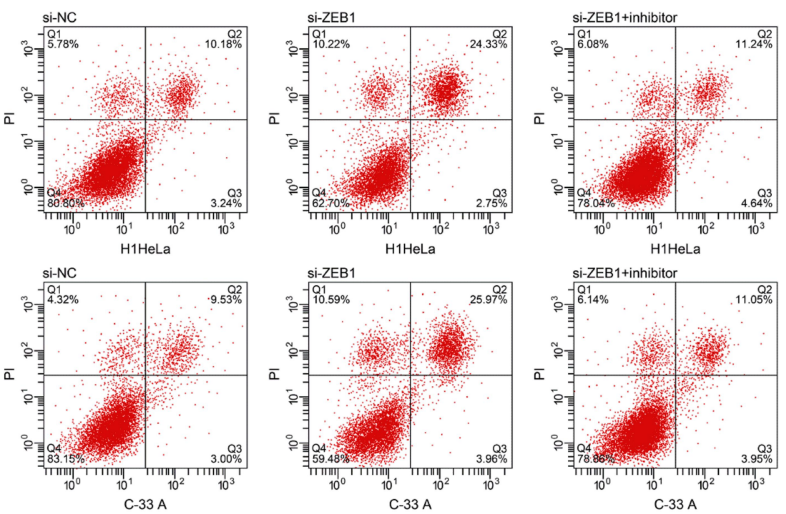

Figure 5 Effects of down-regulating miR-I39-5p on the anti-tumor activity of si-ZEBI in CC cells. (A-D) Inhibiting miR-I39-5p could reverse the inhibiting effect of downregulating ZEBI on the malignant proliferation, invasion, and colony formation, and EMT of CC cells. Protein profiling of EMT-related factors. (E) Inhibiting miR-I39-5p could eliminate the induction of si-ZEBI on the apoptosis of CC cells. Cell flow cytometry profiling. (F) Inhibiting miR-/39-5p could eliminate the induction of si-ZEBI on proapoptotic factors (Caspase-3 and Bax) and the inhibition of it on anti-apoptotic factor (Bcl-2).

Notes: In terms of inter-group comparison or comparison with miR-NC, *Indicates $P<0.05$ and **Indicates $P<0.01$; in terms of comparison with miR-I39-5p, Indicates $P<0.01$.

Abbreviations: miR, microRNA; ZEBI, zinc finger E-box-binding homeobox I; CC, cervical cancer; EMT, epithelial-mesenchymal transition; si, short interfering; NC, negative control; PI, propidium iodide; Bax, Bcl-2-associated X protein; Bcl-2, B-cell lymphoma-2.

A

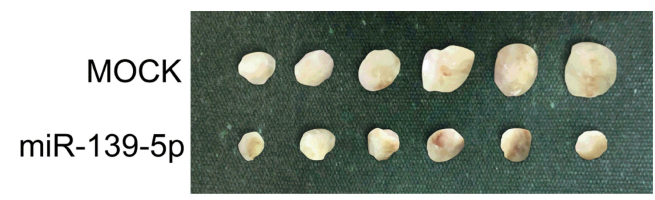

B

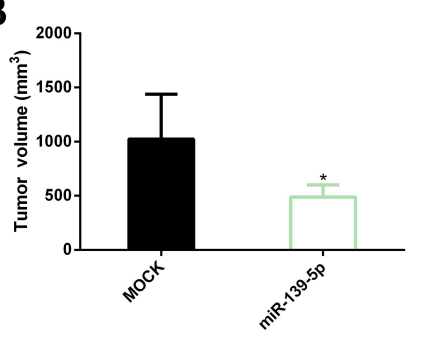

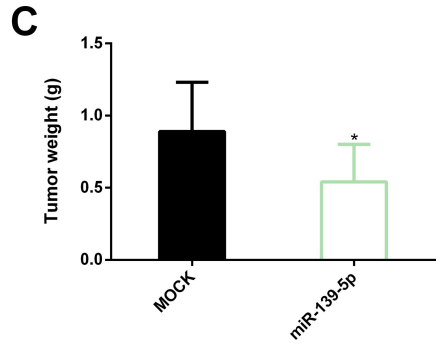

Figure 6 Effect of up-regulating miR-139-5p on tumor growth in CC xenotransplantation model mice. (A) Tumor experimental map of CC xenotransplantation model mice in the two groups. (B, C) Up-regulation of miR-139-5p could inhibit tumor volume and mass in CC xenotransplantation model mice.

Note: *Indicates $\mathrm{P}<0.05$ vs. the MOCK group.

Abbreviations: miR, microRNA; CC, cervical cancer.

expression of pro-apoptotic factors (Caspase-3 and Bax) and decreasing the expression of anti-apoptotic factor Bcl2. Further exploration on the mechanism of miR-139-5p revealed that it had a targeted relationship with $Z E B 1$, and could negatively regulate the level of $Z E B 1$, and downregulating $Z E B 1$ could suppress the aforementioned 
malignant biological behaviors of $\mathrm{CC}$ cells and induce apoptosis. $Z E B 1$ has also been found to be carcinogenic in a variety of gynecological tumors. For instance, Chen et $\mathrm{al}^{35}$ have reported that $Z E B 1$ can promote the accumulation of tumor-related macrophages in the hypoxic tumor microenvironment, thus aggravating the malignant progression of $\mathrm{CC}$ Cui et $\mathrm{al}^{36}$ have also reported that $Z E B 1$ is related to the resistance of ovarian cancer cells against chemotherapy, and targeted regulation of $Z E B 1-S L C 3 A 2$ is conducive to strengthening cisplatin-related chemosensitivity. We downregulated the two indexes meantime, and found that downregulating $m i R-139-5 p$ could strongly eliminate the antitumor activity after down-regulation of $Z E B 1$. Namely, the inhibitory effect of transfecting si-ZEBI on the malignant progression of $\mathrm{CC}$ cells was greatly weakened. All data suggest that $m i R-139-5 p$ can ameliorate the malignant procession of CC cells through targeted inhibition on $Z E B 1$, and the miR-139-5p-ZEB1 axis had molecular regulatory influence on the growth, invasion, as well as EMT of CC cells. Finally, we also found through xenotransplantation assay that up-regulation of $m i R-139-5 p$ could inhibit the growth of tumor in $\mathrm{CC}$ mice, namely reducing the volume and mass of tumor, which indicated that over-expression of miR-139-5p had a positive effect on inhibiting the development of tumor in $\mathrm{CC}$ mice. Based on the above research results, we believe that the development of preparations for targetedly overexpressing $m i R-139-5 p$ or $Z E B 1$ inhibitors may be helpful to the treatment of patients with CC.

Our study has confirmed the functional expression of $m i R-139-5 p-Z E B 1$ in $\mathrm{CC}$, but it still has some room for improvement. First of all, we can supplement the relevant research on whether $m i R-139-5 p$ has upstream factors in the regulatory mechanism of $\mathrm{CC}$ to further expand the molecular regulatory network. In addition, we can also explore the effect of miR-139-5p on miR-139-5p-ZEB1 signal transduction pathway by analyzing whether the molecular action of the pathway has reverse influence on miR-139-5p. Furthermore, we can analyze the influence of $m i R-139-5 p$ on the chemical sensitivity of CC and explore whether $m i R$ $139-5 p$ is helpful to alleviate the drug resistance of patients. Finally, we can supplement the detection of inflammatory factors to further explore the effect of $m i R-139-5 p$ on inflammation in pathological process of $C C$. In the future, we will gradually improve our research from the above points.

To sum up, we have proposed for the first time that $m i R-139-5 p-Z E B 1$ axis can exert molecular regulation on the growth, invasion, and EMT of $\mathrm{CC}$, which may provide a new direction for treating CC.

\section{Disclosure}

The authors report no conflicts of interest for this work.

\section{References}

1. Kim H, Lee KK, Heo MH, Kim JY. The prognostic factors influencing overall survival in uterine cervical cancer with brain metastasis. Korean J Intern Med. 2019;34(6):1324-1332.

2. Zhang Y, Guo X, Wang G, et al. Real-world study of the incidence, risk factors, and prognostic factors associated with bone metastases in women with uterine cervical cancer using surveillance, Epidemiology, and End Results (SEER) data analysis. Med Sci Monit. 2018;24:6387-6397. doi:10.12659/MSM.912071

3. Arbyn M, Weiderpass E, Bruni L, et al. Estimates of incidence and mortality of cervical cancer in 2018: a worldwide analysis. Lancet Glob Health. 2020;8(2):e191-e203. doi:10.1016/S2214-109X(19) 30482-6

4. Yao S, Xu J, Zhao K, et al. Down-regulation of HPGD by miR-146b$3 p$ promotes cervical cancer cell proliferation, migration and anchorage-independent growth through activation of STAT3 and AKT pathways. Cell Death Dis. 2018;9(11):1055. doi:10.1038/ s41419-018-1059-y

5. Park S, Eom K, Kim J, et al. MiR-9, miR-21, and miR-155 as potential biomarkers for HPV positive and negative cervical cancer. BMC Cancer. 2017;17(1):658. doi:10.1186/s12885-017-3642-5

6. Liu S, Song L, Zeng S, Zhang L. MALAT1-miR-124-RBG2 axis is involved in growth and invasion of HR-HPV-positive cervical cancer cells. Tumour Biol. 2016;37(1):633-640. doi:10.1007/s13277-0153732-4

7. Xin M, Qiao Z, Li J, et al. miR-22 inhibits tumor growth and metastasis by targeting ATP citrate lyase: evidence in osteosarcoma, prostate cancer, cervical cancer and lung cancer. Oncotarget. 2016;7 (28):44252-44265. doi:10.18632/oncotarget.10020

8. Qu ZY, Cui GY, Shi PJ, Wang HQ. Potential suppressive functions of microRNA-504 in cervical cancer cells malignant process were achieved by targeting PAICS and regulating EMT. Arch Gynecol Obstet. 2020;302(1):173-182. doi:10.1007/s00404-020-05538-x

9. Zong ZH, Liu Y, Chen S, Zhao Y. Circ_PUM1 promotes the development of endometrial cancer by targeting the miR-136/NOTCH3 pathway. J Cell Mol Med. 2020;24(7):4127-4135. doi:10.1111/ jcmm.15069

10. Wu N, Song H, Ren Y, Tao S, Li S. DGUOK-AS1 promotes cell proliferation in cervical cancer via acting as a ceRNA of miR-653-5p. Cell Biochem Funct. 2020.

11. Ji X, Guo H, Yin S, Du H. miR-139-5p functions as a tumor suppressor in cervical cancer by targeting TCF4 and inhibiting Wnt/ beta-catenin signaling. Onco Targets Ther. 2019;12:7739-7748. doi:10.2147/OTT.S215796

12. Li J, Li Q, Lin L, et al. Targeting the Notch1 oncogene by miR-139$5 p$ inhibits glioma metastasis and epithelial-mesenchymal transition (EMT). BMC Neurol. 2018;18(1):133.

13. Fang J, Huang C, Ke J, et al. lncRNA TTN-AS1 facilitates proliferation, invasion, and epithelial-mesenchymal transition of breast cancer cells by regulating miR-139-5p/ZEB1 axis. J Cell Biochem. 2020. doi: $10.1002 /$ jcb. 29700

14. Yang C, Sun J, Liu W, et al. Long noncoding RNA HCP5 contributes to epithelial-mesenchymal transition in colorectal cancer through ZEB1 activation and interacting with miR-139-5p. Am J Transl Res. 2019;11(2):953-963.

15. Yue S, Wang L, Zhang H, et al. miR-139-5p suppresses cancer cell migration and invasion through targeting ZEB1 and ZEB2 in GBM. Tumour Biol. 2015;36(9):6741-6749. doi:10.1007/s13277-015-3372-8

16. Xu J, Wang $\mathrm{H}$, Wang $\mathrm{H}$, et al. The inhibition of miR-126 in cell migration and invasion of cervical cancer through regulating ZEB1. Hereditas. 2019;156:11. 
17. Gan L, Chen Y, Liu H, Ju WH. Long non-coding RNA ZEB1-antisense 1 affects cell migration and invasion of cervical cancer by regulating epithelial-mesenchymal transition via the p38MAPK signaling pathway. Gynecol Obstet Invest. 2019;84 (2):136-144. doi:10.1159/000493265

18. Kuhn L, Saidu R, Boa R, et al. Clinical evaluation of modifications to a human papillomavirus assay to optimise its utility for cervical cancer screening in low-resource settings: a diagnostic accuracy study. Lancet Glob Health. 2020;8(2):e296-e304. doi:10.1016/ S2214-109X(19)30527-3

19. Canaz E, Ozyurek ES, Erdem B, et al. Preoperatively assessable clinical and pathological risk factors for parametrial involvement in surgically treated FIGO stage IB-IIA cervical cancer. Int J Gynecol Cancer. 2017;27(8):1722-1728. doi:10.1097/IGC.0000000000001060

20. Liu M, Wang Z, Liu Q, Zhu H, Xu N. Expression of micro-RNA-492 (MiR-492) in human cervical cancer cell lines is upregulated by transfection with wild-type P53, irradiation, and 5-fluorouracil treatment in vitro. Med Sci Monit. 2018;24:7750-7758. doi:10.12659/ MSM.911585

21. Msyamboza KP, Phiri T, Sichali W, Kwenda W, Kachale F. Cervical cancer screening uptake and challenges in Malawi from 2011 to 2015: retrospective cohort study. BMC Public Health. 2016;16 (1):806. doi:10.1186/s12889-016-3530-y

22. Rui X, Xu Y, Jiang X, Ye W, Huang Y, Jiang J. Long non-coding RNA C5orf66-AS1 promotes cell proliferation in cervical cancer by targeting miR-637/RING1 axis. Cell Death Dis. 2018;9(12):1175. doi:10.1038/s41419-018-1228-z

23. Babion I, Jaspers A, van Splunter AP, van der Hoorn IAE, Wilting SM, Steenbergen RDM. miR-9-5p exerts a dual role in cervical cancer and targets transcription factor TWIST1. Cells. 2019;9:1. doi:10.3390/cells9010065

24. Gu X, Dong M, Liu Z, Yang J, Shi Y. MiR-499a-5p inhibits proliferation, invasion, migration, and epithelial-mesenchymal transition, and enhances radiosensitivity of cervical cancer cells via targeting eIF4E. Onco Targets Ther. 2020;13:2913-2924. doi:10.2147/OTT. $\mathrm{S} 241631$

25. Miyoshi J, Toden S, Yoshida K, et al. MiR-139-5p as a novel serum biomarker for recurrence and metastasis in colorectal cancer. Sci Rep. 2017;7:43393. doi:10.1038/srep43393
26. Wang Y, Li J, Xu C, Zhang X. MicroRNA-139-5p inhibits cell proliferation and invasion by targeting RHO-associated coiled-coilcontaining protein kinase 2 in ovarian cancer. Oncol Res. 2018;26 (3):411-420. doi:10.3727/096504017X14974343584989

27. Sun C, Sang M, Li S, et al. Hsa-miR-139-5p inhibits proliferation and causes apoptosis associated with down-regulation of c-Met. Oncotarget. 2015;6(37):39756-39792. doi:10.18632/oncotarget.5476

28. Catanzaro G, Besharat ZM, Miele E, et al. The miR-139-5p regulates proliferation of supratentorial paediatric low-grade gliomas by targeting the PI3K/AKT/mTORC1 signalling. Neuropathol Appl Neurobiol. 2018;44(7):687-706.

29. Yang B, Zhang W, Sun D, et al. Downregulation of miR-139-5p promotes prostate cancer progression through regulation of SOX5. Biomed Pharmacother. 2019;109:2128-2135. doi:10.1016/j. biopha.2018.09.029

30. Zhao YL, Li JB, Li YJ, Li SJ, Zhou SH, Xia H. Capn4 promotes esophageal squamous cell carcinoma metastasis by regulating ZEB1 through the Wnt/beta-catenin signaling pathway. Thorac Cancer. 2019;10(1):24-32. doi:10.1111/1759-7714.12893

31. Qin Y, Yu J, Zhang M, Qin F, Lan X. ZEB1 promotes tumorigenesis and metastasis in hepatocellular carcinoma by regulating the expression of vimentin. Mol Med Rep. 2019;19(3):2297-2306.

32. Murata M, Ito T, Tanaka Y, Yamamura K, Furue K, Furue M. OVOL2-mediated ZEB1 downregulation may prevent promotion of actinic keratosis to cutaneous squamous cell carcinoma. J Clin Med. 2020;9(3):618. doi:10.3390/jcm9030618

33. Liu J, Li C, Jiang Y, Wan Y, Zhou S, Cheng W. Tumor-suppressor role of miR-139-5p in endometrial cancer. Cancer Cell Int. 2018;18:51. doi:10.1186/s12935-018-0545-8

34. Liu X, Li Y, Wen J, Qi T, Wang Y. Long non-coding RNA TTN-AS1 promotes tumorigenesis of ovarian cancer through modulating the miR-139-5p/ROCK2 axis. Biomed Pharmacother. 2020;125:109882. doi:10.1016/j.biopha.2020.109882

35. Chen XJ, Deng YR, Wang ZC, et al. Hypoxia-induced ZEB1 promotes cervical cancer progression via CCL8-dependent tumour-associated macrophage recruitment. Cell Death Dis. 2019;10(7):508. doi:10.1038/s41419-019-1748-1

36. Cui Y, Qin L, Tian D, et al. ZEB1 promotes chemoresistance to cisplatin in ovarian cancer cells by suppressing SLC3A2. Chemotherapy. 2018;63(5):262-271. doi:10.1159/000493864
Cancer Management and Research

\section{Publish your work in this journal}

Cancer Management and Research is an international, peer-reviewed open access journal focusing on cancer research and the optimal use of preventative and integrated treatment interventions to achieve improved outcomes, enhanced survival and quality of life for the cancer patient.
The manuscript management system is completely online and includes a very quick and fair peer-review system, which is all easy to use. Visit http://www.dovepress.com/testimonials.php to read real quotes from published authors. 